\title{
Vereinnahmung von Geschichte und Literatur durch rumänische Kulturpolitik. Die Kronstädter Publikation Karpatenrundschau (1968-1970)
}

\section{Delia COTÂRLEA}

Lekt. Dr., Transilvania-Universität Kronstadt / Braşov.

E-Mail: delia.cotarlea@unitbv.ro

\begin{abstract}
The present study takes two tendencies into account that have shaped the cultural contact between the Romanian culture and the culture of the German minority in Romania. On the one hand, the re-writing of history respectively of the historical discourse according to cultural policy of the Romanian communist state is envisaged, on the other hand, the selection of articles on Romanian culture and literature published in the weekly Karpatenrundschau are analysed in order to trace tendencies cultural transfer.
\end{abstract}

Key-words: re-writing of historical discourse, cultural policy during the communist regime

\section{Einleitung}

In der vorliegenden Untersuchung werden zwei Tendenzen berücksichtigt, die den Kulturkontakt zwischen der rumänischen Mehrheit und deutschen Minderheit in Rumänien geprägt haben. Einerseits wird die Vereinahmung der Geschichte bzw. des historischen Diskurses durch die Kultupolitik des rumänischen sozialistischen Staates ins Auge gefasst, andererseits die Auswahl der in der Kronstädter Wochenschrift Karpatenrundschau veröffentlichten Artikel zur rumänischen Kultur und Literatur betrachtet, um mögliche Tendenzen in der damaligen kulturellen Dynamik aufzuzeigen. Es wird dabei analysiert, ob und 
inwieweit die Themen- und Textauswahl Rückschlüsse auf die Kultupolitik des rumänischen Staates, möglicherweiser auf die Vorgehensweise der Redaktion zulassen.

Gleich nach dem Ende des Zweiten Weltkrieges durchläuft Rumänien aufgrund der Sowjetisierung eine antinationale Phase. Der Klassenkampf gilt als Motor der Entwicklung der kommunistischen Gesellschaft. ${ }^{1}$ Erst Mitte der Sechzigerjahre, als ein politisches „Tauwetter“ einsetzt, ist das Bekenntnis zum Nationalgefühl wieder möglich. Das nationale Thema gewinnt auf diskursiver Ebene nicht aufgrund patriotischer Gefühle an Bedeutung, sondern in der Absicht, sich von der sowjetischen Vorherrschaft loszulösen und ein selbständiges Modell kommunistischer Diktatur zu gründen. Das bis dahin propagandistische slawische Element verblasst, ,eine langsame, aber stetige Entwicklung, die 1964 in der berühmten Unabhängigkeitserklärung der Rumänischen Arbeiterpartei kulminiert [... $]^{\text {c2 }_{2}}$ macht sich bemerkbar. Der rumänische Kommunismus wendet sich zum Nationalismus, kehrt dem Internationalismus den Rücken, ,eine Wende, zumindest was die Rhethotik anbelangt, von 180 Grad! “" 3 Dadurch lässt sich ab Mitte der Sechzigerjahre die Zunahme der Beschäftigungen mit der eigenen Geschichte und Literatur erklären, der politische Rahmen erlaubt und steuert die (Selbst)Darstellungen sowohl der Mehrheit als auch der nun sogenanten mitwohnenden Nationalitäten, eigentlich ein systemkonformer Terminus für Minderheiten, die unter der oben angeführten Bezeichnung als Teile des Systems fungieren sollen. Es setzt eine Etappe der Auseinandersetzung mit der Geschichte, Kultur und Literatur des sogenannten ,eigenen“ Landes ein, durch die eine „Isolation der Utopie“" beabsichtigt wird. Die mittelmäßige Existenz im Kommunismus

1 Boia, Lucian: Geschichte und Mythos. Über die Gegenwart des Vergangenen in der rumänischen Gesellschaft. Köln 2003, S. 87.

2 Boia 2003, S. 89.

3 Ebd.

4 Ebd., S. 90. 
kann nur durch nationalistische Rhetorik überbrückt werden, denn dadurch ist es möglich, ,der bedrückenden Realität zu entfliehen."

Ab 1971, dem Jahr der rumänischen „Kulturrevolution“, setzt eine zunehmende Verhärtung ein, die Isolation der Utopie wird ad absurdum weitergeführt, nationale Ideale und Klassenkampf bestimmen den öffentlichen Diskurs:

Der Kommunismus gedieh als Amalgam von authentischen Traditionen und kommunistischen Zielen. Die kommunistische Diktatur bediente sich seiner als Macht- und Legitimationsinstrument. Was eine Aufwertung der Vergangenheit schien, war nichts als Manipulation. [...] - hier nationale Ideale, dort soziale Konflikte [...] Die kommunistische Dialektik war imstande, jede Art von Gegensätzen zu harmonisieren. ${ }^{6}$

Wenn nach großen Taten gefragt wird, liefert sie der Diskurs großzügig nach. Man macht allerhand Spagate, von der Gegenwart in die Zwischenkriegszeit, über das Mittelalter bis in die Frühgeschichte, um den Nationalismus zu stärken und den Klassenkampf zu rechtfertigen. ${ }^{7}$

In diesem kulturpolitischen Kontext der Liberalisierung wird 1968 die regionale Wochenschrift Karpatenrundschau als Nachfolgerin der anfangs linientreuen Publikation Volkszeitung gegründet. Die Volkszeitung (1957-1968) dokumentiert in den ersten Jahren fast ausschließlich die Erfolge der ,sozialistischen Aufbaufront" und ,wiederkäut in klassenkämpferischem Ton die Vorgaben und Losungen der Regierungspartei." ${ }^{8}$ Allmählich finden aber auch Schwerpunkte wie Kultur und Geschichte der deutschen Minderheit Platz auf ihren Seiten.

5 Ebd.

${ }^{6}$ Ebd., S. 92.

7 Ebd., S. 93-97.

${ }^{8}$ Vgl. Schuster, Hannes: Ein Ort kollektiver Selbstvergewisserung. In: Siebenbürgische Zeitung vom 28.3.2017. https://www.siebenbuerger. de/zeitung/artikel/kultur/6664-ein-ort-kollektiver.html (Abruf am 4.9.2018). 
Berichterstattungen zu historischen Themen werden nicht zufällig behandelt, denn in ,den offiziellen Verlautbarungen zur ,vaterländischen Geschichte' klammerte das national-kommunistische Rumänien die historische Existenz seiner Minderheiten entweder völlig aus oder spielte sie bis zur Bedeutungslosigkeit herunter.“9

Die Karpatendrundschau wird in einer „Tauwetterperiode“ ins Lebens gerufen, möchte durch ihr Profil den Aufschwung Rumäniens dokumentieren. Durch ihre Beiträge setzt sie sich vor allem zum Ziel, „nach bestem Wissen und Können in den fruchtbaren Meinungsaustausch, der heute zur Notwendigkeit wurde, sich einzuschalten, will aktiv mithelfen, das gesellschaftliche, politische und kulturelle Bild unseres Vaterlandes $\mathrm{zu}$ formen." ${ }^{10}$ Das Programm der neuen (über)regionalen Wochenschrift für Gesellschaft, Politik und Kultur ist klar formuliert - linientreu, gleichzeitig aber auch im Versuch, den Anliegen der Minderheit gerecht zu werden: ein komplizierter Spagat, um Heterogenitäten zu überbrücken. Vielleicht war die damalige Karpenrundschau, nach heutigem Geschmack, etwas zu vielseitig ausgerichtet, denn man kann außer den aktuellen politischen und gesellschaftlichen Nachrichten, vieles über Kultur, Literatur, Theater und Film sowie Kunst, Sport und Wissenschaft, aber auch überAlltagsthemen, wieHaushaltstipps, Mode, Männer- und Frauengewohneiten lesen. ${ }^{11}$ Vom Format und von der Aufstellung her passt sich die Karpatenrundschau an die systemkonformen Vorgaben an. Auf den ersten Seiten wird dem politischen Regime mit seinen Fortschritten Aufmerksamkeit geschenkt, die anderen sind der Minderheit gewidmet.

9 Ebd.

${ }^{10}$ o.V.: Liebe Leser! In: KR 1/1968, S. 1.

${ }^{11}$ Vgl. Puchianu, Carmen / Cotârlea Delia: Zwischen politischem Kompromiss und ästhetischem Anspruch: Rumäniendeutsche Lyrik der 1980er und 1990er Jahre im Spiegel der Kronstädter Wochenschrift Karpatenrundschau Teil I. In: Germanistische Beiträge 42/2018, S. 48-64. 
Durch die kulturpolitische Verschärfung ab Mitte der Siebzigerjahre und die zunehmende Ausreise der Deutschstämmigen schrumpft die Publikation zusammen. Nach der „Wende“ bleiben von den 16 Seiten nur noch 8 übrig, ab 1993 erscheint die $K R$ als Beilage der Allgemeinen Deutschen Zeitung für Rumänien.

\section{Vereinnahmung der Geschichte durch nationalistische Kulturpolitik}

Nach der Untersuchung der Wochenschrift Karpatenrundschau im Zeitraum 1968-70 kann eine Doppelbödigkeit des öffentlichen historischen Diskurses festgestellt werden. Einerseits beschäftigt man sich mit der eigenen Minderheitengeschichte, da dies nach längerer Zeit gutgeheißen wird, andererseits vereinnahmt das politische System den historischen Diskurs durch seine eigene Rhetorik. Beispielsweise wird der Terminus Mitwohnende Nationalität konsequent verwendet. Auf den ersten Seiten der Wochenschrift wird meist ein inszeniertes Miteinanderleben im Kommunismus vorgetäuscht, es geht im offiziellen Diskurs um ,,aus dem Zustand der Zersplitterung zu einer Nation der Einheit zusammenzuwachsen, [... $]^{\text {"12 }}$, um „Brüderlichkeit [...] zum rumänischen Volk ${ }^{\text {“13 }}$, um „Solidarität ${ }^{\text {" }}$ des Proletariats“"14, um die „Kulturschaffenden deutscher Nationalität ${ }^{\text {"15}}$. Die Bedeutung der rumänischen Geschichte wird propagandistisch in zahlreichen Artikeln hervorgehoben, Minderheiten und Mehrheit werden durchgängig als Einheit verstanden. Lucian Boia behauptet, in der kommunistischen Zeit sei der Mythos der Einheit auf seinem Höhepunkt gewesen, die unerschüttliche Einheit sei zu einem Wesensmerkmal

${ }^{12}$ Liebhardt, Franz: Geschichte und Aktualität. In: KR 27/1968, S. 1-2.

${ }^{13}$ Ebd., S. 2.

${ }^{14}$ Vgl. Cotot, Ion: Solidarität. In: KR 9/1968, S. 1.

${ }^{15}$ Vgl. o. V.: Beratung beim ZK der RKP mit Wissenschaftlern und Kulturschaffenden deutscher Nationalität. In: KR 20/1968, S. 1. 
der Rumänen geworden, denn diese Art von Diskurs entspreche dem Ideal der Diktatur, eine uniformierte Gesellschaft zu schaffen. Es handelt sich dabei keineswegs um Patriotismus, sondern um propagandistische Ideologie. Dadurch wird der historische Diskurs der Minderheit von den politisch-ideologischen Vorgaben geprägt und geändert. Kein wichtiges Ereignis bleibt verschont, sogar das kartographische Werk des siebenbürgischen Humanisten Johannes Honterus, Rudimenta cosmographica, wird von dem sogenannten "Dakischen Plan" ${ }^{16}$ vereinnahmt. Der siebenbürgische Reformator habe nämlich „die territoriale und historische Einheit der Rumänischen Lande, des antiken Daziens, wiederholte Male unterstrichen. ${ }^{\text {"17 }}$ Unter der 1542 entworfenen Karte steht die Erklärung: „Die älteste Landkarte des heutigen Rumäniens. [sic!]"'18

Das heterogene Mittelalter wird ebenfalls auf einen gemeinsamen Nenner gebracht. Die Involvierung sächsischer Jobagendörfer im antifeudalen Kampf wird in einen Klassenkampf uminterpretiert die Rumänen werden als Urbevölkerung bezeichnet. ${ }^{19}$ Um Einheit geht es auch in der Wiedergabe des historischen Moments 1848, was Lucian Boia als eine grobe Fälschung betrachtet, denn es habe kein grenzübergreifendes einheitlichens revolutionäres Programmm gegeben. Außerdem wird dieses Ereignis ebenfalls nach dem Ansatz des Klassenkampfes neu gedeutet.

Die Gründung Großrumäniens als Vereinigung der rumänischen Lande dient dazu, zumindest aus rhetorischer Sicht, den Mythos der rumänischen Einheit zu untermauern. In der $K R$ wird die allgemeine Solidarität der deutschen Minderheiten im Hinblick auf die Vereinigung mit Rumänien betont, die Gründung

${ }^{16}$ Boia 2003, S. 164.

${ }^{17}$ Binder, Paul: Die Dazienvision des Johannes Honterus. In: KR 21/1968, S. 6.

${ }^{18}$ Ebd.

${ }^{19}$ Kroner, Michael: Jobagendörfer in Siebenbürgen. In: KR 26/1969, S. 7. 
Großrumäniens sei kein spontaner Entschluss gewesen, ,ist dem jahrhundertlangen Zusammenleben und gemeinsamen Kampf des rumänischen Volkes und der mitwohnenden Nationalitäten gegen soziale und nationale Unterdrückung, gegen alles Rückständige verwurzelt. “" ${ }^{20}$ Dieses grundlegende politische Ereignis wird wiederkäuerisch durch politische Losungen auf den sozialistischen Alltag übertragen: Die nationale Frage sei durch den marxistisch-leninistischen Ansatz gelöst, durch den Sozialismus sei die Einheit und Geschlossenheit erreicht worden.

Nach 1945 gibt es nurnoch die mitwohnenden Nationalitäten, die zum Aufbau des Kommunismus beitragen:

Es bedarf wohl kaum einer besonderen Beweisführung, dass zusammen mit dem rumänischen Volk auch die mitwohnenden Nationalitäten und unter ihnen die mit diesem schönen, reich gesegneten, zutiefst heimatlichen Land tausenfällig verwachsenen deutschen Bevölkerungsteile Siebenbürgens und des Banats von den lebensvollen, kraftspendenden Zusammenhängen zwischen Geschichte und Aktualität miteingeschlossen sind. ${ }^{21}$

Der Mythos der Einheit aller Nationalitäten auf dem Gebiet der Volksrepublik bzw. des Sozialistischen Rumäniens wird bis Ende des Regimes zum Paroxymus getrieben.

Abschließend lässt sich bemerken, dass die Vereinnahmung der Geschichte durch den Kommunismus schon in den Anfangsjahren der $K R$ nachgezeichnet werden kann. Weiterhin verdeutlicht der Nationalimus die Nivellierungs- und Assimilationstendenzen in Ceaușescus Minderheitenpolitik.

${ }^{20}$ Zimann, Mihai: Die Schwaben und die Vereinigung. In: KR 41/1968, S. 8.

${ }^{21}$ Liebhard, Franz: Geschichte und Aktualität. In: KR 27/1968, S. 1-2. 


\section{Rumänische Literatur in der Karpatenrundschau (1968-70)}

In den ersten drei Jahren (1968-70) kann eine stetige Zunahme rumänischer Literatur in der Karpatenrundschau festgestellt werden. 1968 ist eine geringe Anzahl von Texten vertreten, ihre Zahl verdreifacht sich 1969 und steigt im Jahr 1970 weiter. Nach der Textauswahl zu urteilen, führen wir den kontinuierlichen Zuwachs einerseits auf die Vereinnahmnung der Literatur durch ideologiekonforme Texte zurück, die in jeder journalistischen Publikation in Rumänien gedruckt werden müssen, andererseits auf die Liberalisierung Ende der Sechzigerjahre, die Freiraum für Texte schafft, die den ästhetischen Kriterien genügen. Im Jahr 1968 werden sowohl tradierte als auch rehabilitierte Autoren publiziert - Barbu Delavrancea, Vasile Rebreanu, Eugen Ionescu. Es erscheinen auch literaturkritische Beiträge zu Leben und Werk rumänischer Autoren, beispielsweise zu Ion Pillat. 22 Jüngere Autoren sind marginal durch Nina Cassian und Sînziana Pop vertreten.

Einige in der Karpatenrundschau veröffentlichten literaturkritischen Aufsätze zur rumänischen Literatur beziehen sich sowohl auf linientreue als auch auf authentische, unpolitische Literatur. Ein Interview mit dem heutzutage wenig bekannten Dichter Corneliu Sturzu, zum damaligen Zeitpunkt Lektor am Lehrstuhl für Weltliteratur der Universität Iași, zeigt, wie wichtig es für die traditionsreiche literarische Szene aus Iași gewesen sei, wieder im Mittelpunkt zu stehen, wenn das auch bedeutet, sich dem systemkonformen Diskurs anzupassen. Eine neue Generation von Lyrikern wird angeführt, aus heutiger Sicht eher zweitrangige Dichter, darunter aber auch Namen, die sich nach ästhetischen Kriterien durchgesetzt haben, beispielsweise Dan Laurențiu. Deklarativ wird die Förderung junger Talente

${ }^{22}$ Wittstock, Joachim: Zwischen Tradition und Erneuerung. In: KR 13/1968, S. 8-9. 
angestrebt, jedoch unter der Voraussetzung ideologischer Anpassung: „Die junge Schriftstellergeneration versucht sich oft erfolgreich an den großen Zeitproblemen, ist bemüht, die Fragen der Gegenwart im Geiste der Zeit zu beantworten." ${ }^{23}$

Ein Band zur Geschichte der rumänischen Literatur wird ausgiebig vorgestellt. Er dient als ,eine wertvolle Hilfe für den literarisch Interessierten" und leistet einen grundlegenden Beitrag „zur Vervollständigung eines als sehr notwendig empfundenen Standardwerkes über rumänische Literatur" ${ }^{24}$

Im Zeitraum 1969-70 fächert sich die Bandbreite der in der KR publizierten rumänischen Literatur auf. Nicht nur eine erhebliche Zunahme sondern auch eine Differenzierung des Literaturangebots kann festgestellt werden. Veröffentlicht werden weiterhin tradierte, rehabilierte Autoren - Nicolae Balotă, Lucian Blaga, Tudor Arghezi, Mihai Eminescu, I. C. Caragiale, Camil Petrescu, Mihail Sadoveanu - wie auch die „Hofpoeten“ Alexandru Andrițoiu und Fănuș Neagu. Dem 1967 verstobenen Tudor Arghezi wird besondere Aufmerksamkeit geschenkt, was sich auch dadurch erklären lässt, dass sich der Lyriker nach seiner Rehabilitierung dem politischen System gegenüber loyal zeigt und ideologiekonforme Texte liefert. Gegen Ende seines Lebens wird Arghezi sogar zum nationalen Dichter gekürt. Nicolae Balotă ist ein ähnlicher Fall: Nachdem er 1963 mit der Securitate ein Abkommen unterzeichnet, wird er aus langjähriger Haft entlassen und gilt als sauberer Dichter. Sadoveanu ist in den Fünfzigerjahren ebenfalls zu einem linientreuen Dichter geworden. Paradoxerweise können Texte obiger Autoren veröffentlicht werden, ohne dabei ausschließlich der kulturpolitischen Schiene zu folgen: Unter dem Vorwand, es handle sich um vom Sozialismus überzeugte Dichter, können auch unpolitische Texte erscheinen. Erneut zeigt sich die Vereinnahmung aller Lebensbereiche durch den diktatorischen Staat.

${ }^{23}$ Schuller, Hans: Das literarische Jassy. In: KR 20/1968, S. 1 und 5.

${ }^{24}$ Anger, Horst: Synthese und Beispiel. In: KR 31/1968, S. 9. 
In der Zeitspanne 1969-70 machen sich die positiven Wirkungen des politischen „Tauwetters“ bemerkbar. Die Zahl der in der Karpatenrundschau publizierten jüngeren nimmt Autoren zu, Texte von Nina Cassian, Nicolae Breban, Ana Blandiana, Marin Sorescu, D.R. Popescu werden veröffentlicht. Die Kronstädter Publikation zeigt auch für jüngere, lokale Dichter Interesse. Gedichte des damals 36-jährigen Kronstädters Ion Popescu Topolog werden in die Karpatenrundschau aufgenommen, 1970 ist ein weiterer lokaler Schriftsteller, Cuza Constantin, mit Texten vertreten.

$\mathrm{Zu}$ den neuesten Entwicklungen in der rumänischen Lyrik heißt es in der Kurzanalyse von Voicu Bugariu Kühne Reflexionen. Die rumänische Gegenwartslyrik: „In den 60er Jahren brach für die rumänische Lyrik eine überaus fruchtbare Periode an. Bezeichnend war die Bemühung der Dichter um eine Erneuerung der lyrischen Ausdrucksweise und der experimentelle Charakter ihrer Lyrik. ${ }^{25}$ Es geht in dem Bericht von Bugariu um Literatur, die den ästhetischen Ansprüchen der Zeit entspricht, die sich von der linientreuen Literatur abgrenzt. Es fallen die Namen Marin Sorescu, Nichita Stănescu, Ana Blandiana, Cezar Baltag, Leonid Domiv, Radu Carneci u.a. Ihre Verse, die neuen Gedichte, ,bereichert mit Ausdrucksmitteln der modernen Weltliteratur, heben das eigenartig Rumänische über den streng nationalen Rahmen hinaus. " ${ }^{26}$ Subkutan schwingt im Zitat die Aussage mit, dass die nationale Themaik und nationalistische Ideologie in den Texten der neuen Generation überwunden ist. Um die Texte jedoch ideologisch zu untermauern, wird ihnen ein humanistischer Zug im Sinne rumänischer tradierter Lyrik zugeschrieben.

Zusammenfassend lässt sich sagen, dass in der rumänischer Lyrik der Jahres 68 die Tendenz zum ,zurück zum Klassischen' bemerkbar macht, wobei dieses im Sinne eines Zurück zu den Traditionen

${ }^{25}$ Bugariu, Voicu: Kühne Reflexionen. Die rumänische Gegenwartslyrik.

In: KR 9/1969, S. 7.

${ }^{26}$ Ebd. 
unserer humanistischen klassischen Lyrik aufzufassen ist. Eine verantwortungsvolle, reflexive Haltung ist den meisten dieser Dichter eigen. ${ }^{27}$

In einem späteren Beitrag heißt es im Rückblick auf die junge rumänische Lyrik des Jahres 1969, dass sie sich als ,,verneinenden Lyrismus" beschreiben lasse, sowie eine Vermenschlichung, gerade durch Verneinung erfahre. „Harmonien de Verneinung erzeugen in ihrem Zusammenspiel eine Bejahung. ${ }^{\text {" }}{ }^{28}$ Die neuen Tendenzen in der rumänischen jüngeren Lyrik werden in einem von Horst Schuller geführten Interview mit Ana Blandiana erörtert. „Wichtig ist, dass man sich für all das voll und ganz einsetzt, woran man aus innerster Überzeugung glaubt. Der Vorzug de jungen Generation besteht gerade darin, dass sie nicht Lippenbekenntnisse schreibt, sie kämpft für Ideen von denen sie ergriffen wurde. ${ }^{\text {“29 }}$ Das Zitat belegt die Abgrenzung der jüngeren Dichter von den politisch-ideologischen Vorgaben. Gute Literatur kann nicht in ein ideologisches Korsett gezwängt werden, sie verfehlt auch ihren Zweck, wenn sie eine systemkonforme Erziehungsfunktion erfüllt.

Viele verstehen auch die Forderung, der Dichter müsse die Gesellschaft erziehen, auf eine simple Weise.[...] Gute Literatur entsteht nicht aus einem gut gemeinten pädagogischen Plan heraus, doch sie erzieht immer, wenn sie gut ist.Dichtung sensibilisiert den Menschen, bloss das kann sie erreichen - und das ist schon ungeheuer viel. ${ }^{30}$

Rumänische Literatur wird nicht nur im überregionalen Kontext berücksicht, das Kronstädter literarische Leben wird ebenso ins Auge gefasst. Die Karpatenrundschau veröffentlicht dazu ein Gespräch mit dem damaligen Vorsitzenden der vor Kurzem

${ }^{27}$ Ebd.

${ }^{28}$ Bugariu, Voicu: Freude am Entdecken. Die rumänische Poesie und Prosa des Vorjahres. In: KR 11/1970, S. 8.

${ }^{29}$ Anger, Horst: Dichtung sensibilisiert: Aufzeichnungen aus einem Gespräch mit Ana Blandiana. In: KR 24/1970, S. 8.

${ }^{30}$ Ebd. 
entstandenen Kronstädter Schriftstellervereinigung. ${ }^{31}$ Es geht in der Diskussion um die Umgestaltung der Kronstädter Filiale des Rumänischen Schriftstellerverbandes im Hinblick auf mehr Autonomie. „Die Bildung unserer Schriftstellervereinigung entsprang dem allgemeinen Wunsch, in Südsiebenbürgen einen festen Rahmen für alle begabten Schriftsteller rumänischer,

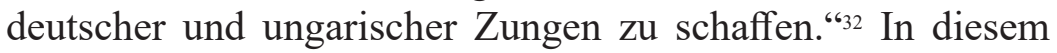
Kontext soll die Schriftstellervereinigung als Hauptspieler der regionalen Kulturarbeit durch Förderung literarischer Kreise, Lesungen, Vortragsabende, Veranstaltungen der Bibliotheken fungieren. Auf deklarativer Ebene wird die Tätigkeit der Schriftstellervereinigung von der nationalen Kulturpolitik vereinnahmt, denn der Zweck ist die Verinheitlichung der Kulturschaffenden aller Nationalitäten. „Das Zusammenleben verschiedener Nationalitäten in Kronstadt und Siebenbürgen gibt dieser Landschaft einen eigenartigen Reiz und fördert außer einer Literatur in rumänischer, deutscher und ungarischer Sprache auch eine vorbildliche Übersetzer- und Vermittlerarbeit." ${ }^{\text {"33 }}$

\section{Themenschwerpunkt Kulturvemittlung in der Karpatenrundschau (1968-70)}

Das Thema der Kulturvermittlung wird konsequent von der Karpatenrundschau aufgegriffen. Adolf Meschendörfers Publikation Die Karpathen als Kulturmittler wird in einem Beitrag behandelt, wobei auf die Bedeutung der Wahrnehmung rumänischer und ungarischer sowie auch der banatschwäbischer Literatur schon für das siebenbürgische Leserpublikum hingewiesen wird. ${ }^{34}$ Der Text hebt Meschendörfers Beitrag zur

${ }^{31}$ o. V.: Kein literarisches Abseits. Gespräch mit Dan Tarchilă, Vorsitzendem der Kronstädter Schriftstellervereinigung. In: KR 35/1970, S. 8-9.

${ }^{32}$ Ebd.

33 Ebd.

${ }^{34}$ Kroner, Michael: Brücke von Bruder zu Bruder. ,Die Karpaten“ Kulturvermittler zwischen den Völkern. In: KR 42/1968, S. 8. 
Völkerverständigung hervor. Außerdem ,schlug [er] durch seine Veröffentlichung die erste Verständigungsbrücke zwischen diesen zwei (Banater und Siebenbürger) wichtigsten Siedlungsgruppen unseres Vaterlandes. ${ }^{{ }{ }^{35}}$

Der Kulturaustausch soll auch anhand der Übersetzungsprozesse veranschaulicht werden. Petru Manolius Erfahrungsbericht So habe ich den "Zauberberg" übersetzt ${ }^{36}$ dokumentiert das Ringen mit der rumänischen Mutter- und der deutschen Sprache, das Pendeln zwischen dem deutschen Gedankengut und der rumänischen Geisteswelt. „Es war ein Gang bis an die Grenzen, wenn man den Gipfel des ,Zauberbergs' tatsächlich erreichen wollte." Der Erfolg des Projektes liege, so Manoliu, in der Kenntnis der sprachlichen Leistungen von M. Eminescu, N. Iorga und M. Sadoveanu. Ein einfaches Übersetzen im Falle des Werkes von Thomas Mann habe sich für Manoliu als nicht ausreichend erwiesen, die Ansiedlung des Zauberbergs in die rumänische Kultur durch die Übertragung sei die wahre Herausforderung gewesen. Den Erfahrungsbericht von Petru Manoliu betrachten wir als besonders wertvoll, da er authentischeAspekte des kulturellen Austausches durch Textübersetzung bearbeitet.

In den nächsten zwei Jahren wird die Bedeutung des Kulturaustausches in der Karpatenrundschau zunehmend thematisiert. Es wird über eine Eminescu-Lenau-Tagung berichtet ${ }^{37}$, die Bezüge zwischen den beiden Dichtern sollen wissenschaftlich von Germanisten von neuen Standpunkten aus untersucht oder ältere Befunde ausgebaut werden. Nicolae Balotă setzt sich wissenschaftlich mit der Rilke-Rezeption in Rumänien, darunter

${ }^{35}$ Kroner, Michael: Menschendörfers „Karpaten“ über die Banater Schwaben. In: KR 19/1968, S. 7.

${ }^{36}$ Manoliu, Petru: So habe ich den „Zauberberg“ übersetzt. In: $K R$ 21/1968, S. 8.

37 Stănescu, Heinz: Eminescu und Lenau. Temeswar 1969 - Tagungsort der internationalen Lenau-Gesellschaft. In: KR 6/1969, S. 9. 
auch mit den wichtigsten Rilke-Übersetzungen, auseinander. Balotă führt eindeutige Beweise an, dass ,rumänische Dichter und Denker in ihm einen grossen exemlarischen Dichter, ja noch mehr: eine Verkörperung der Poesie schlechthin sahen." ${ }^{\text {"38 }}$

Andererseits sind rumänische Publikationen im Ausland ein systemkonformes, nationalstärkendes Thema. Um Kulturaustausch geht es aber in diesen Texten nicht. Es wird versucht, mit Zahlen zu beeindrucken, sie als faktisches Argument in den propagandistischen Diskurs einzubringen: „Allein 1966 wurden 4,2 Millionen Bücher und Broschüren gedruckt. ${ }^{\text {“69 }}$ Man präsentiert die vermeintliche Zunahme des rumänischen Sprachgebrauchs im Ausland:

man spricht im Ausland immer mehr Rumänisch, und zwar handelt es sich nicht bloss um den Gebrauch dieser Sprache beim Verlegen von Büchern und wissenschaftlichen Dokumenten, sondern immer mehr wird Rumänisch auch gelesen und gesprochen dort, wo Fachleute sich mit Fachfragen auseinandersetzen zu haben. [...] Wir denken dabei ganz besonders an Mathematik und Chemie. ${ }^{40}$

Der Texteinschub mit Bezug auf Mathematik und Chemie verdeutlicht die systemkonforme Einstellung, es wird auf die vom System fabrizierten Kompetenzen Elena Ceaușescus im Wissenschaftbereich Chemie angespielt. Andererseits thematisiert der Artikel auch die Notwendigkeit, rumänische wissenschaftliche Werke zu übersetzen, und von Lektoraten für die rumänische Sprache, welche die Aufmerksamkeit auf die rumänische Literatur zu lenken und das Interesse dafür wachzuhalten haben.

Abschließend kann behauptet werden, dass die kommunistische Kulturpolitik alle Ressorts der Karpatenrundschau vereinnahmte. Die Geschichte der Minderheiten wurde nach dem Modell der rumänischen Einheit und des rumänischen

${ }^{38}$ Balotă, Nicolae: Rilke in Rumänien. In: KR 44/1969, S. 8.

39 o. V.: Rumänischer Bucherfolg im Ausland. Unsere Verlage stellen sich vor. In: KR 11/1968.

${ }^{40}$ R., A.: Mehr Rumänisch im Ausland. In: KR 18/1968, S. 8. 
Nationalismus umgedeutet, linientreue rumänische Literatur wurde veröffentlicht. Es bleibt aber auch ein Teil der Texte, die sich im Rahmen des Authentischen, Selbstverständlichen bewegen. Die vom Kommunismus verursachte ideologische, kulturelle und literarische Polarisierung lässt sich in jeder Nummer der Kronstädter Publikation Karpatenrundschau nachzeichnen, die Vereinnahmung von Geschichte, Kultur und Literatur durch das politische Regime ist eklatant.

\section{Literaturverzeichnis}

\section{Sekundärliteratur}

Anger, Horst: Dichtung sensibilisiert: Aufzeichnungen aus einem Gespräch mit Ana Blandiana. In: KR 24/1970, S. 8. Anger, Horst: Synthese und Beispiel. In: KR 31/1968, S. 9. Balotă, Nicolae: Rilke in Rumänien. In: KR 44/1969, S. 8.

Binder, Paul: Die Dazienvision des Johannes Honterus. In: $K R$ 21/1968, S. 6.

Boia, Lucian: Geschichte und Mythos. Über die Gegenwart des

Vergangenen in der rumänischen Gesellschaft. Köln 2003. Bugariu, Voicu: Freude am Entdecken. Die rumänische Poesie und Prosa des Vorjahres. In: KR 11/1970, S. 8.

Bugariu, Voicu: Kühne Reflexionen. Die rumänische Gegenwartslyrik. In: $K R$ 9/1969, S. 7.

Cotot, Ion: Solidarität. In: KR 9/1968, S. 1.

Kroner, Michael: Brücke von Bruder zu Bruder. 'Die Karpaten'

- Kulturvermittler zwischen den Völkern. In: KR 42/1968, S. 8.

Kroner, Michael: Jobagendörfer in Siebenbürgen. In: $K R$ 26/1969, S. 7.

Kroner, Michael: Menschendörfers „Karpaten“ über die Banater Schwaben. In: KR 19/1968, S. 7.

Liebhard, Franz: Geschichte und Aktualität. In: KR 27/1968, S. 1-2. 
Manoliu, Petru: So habe ich den „Zauberberg“ übersetzt. In: KR 21/1968, S. 8.

o. V.: Beratung beim ZK der RKP mit Wissenschaftlern und Kulturschaffenden deutscher Nationalität. In: KR 20/1968, S. 1.

o. V.: Liebe Leser! In: KR 1/1968, S. 1.

o. V.: Rumänischer Bucherfolg im Ausland. Unsere Verlage stellen sich vor. In: KR 11/1968.

o. V.: Kein literarisches Abseits. Gespräch mit Dan Tarchilă, Vorsitzendem der Kronstädter Schriftstellervereinigung. In: KR 35/1970, S. 8-9.

Puchianu, Carmen/Cotârlea Delia: Zwischen politischem Kompromiss und ästhetischem Anspruch: Rumäniendeutsche Lyrik der 1980er und 1990er Jahre im Spiegel der Kronstädter Wochenschrift Karpatenrundschau Teil I. In: Germanistische Beiträge 42/2018, S. 48-64.

R., A.: Mehr Rumänisch im Ausland. In: KR 18/1968, S. 8. Schuller, Hans: Das literarische Jassy. In: KR 20/1968, S. 1 und 5.

Schuster, Hannes: Ein Ort kollektiver Selbstvergewisserung. In: Siebenbürgische Zeitung vom 28.03.2017. https:// www.siebenbuerger.de/zeitung/artikel/kultur/6664-einort-kollektiver.html (Abruf am 4. 9. 2018).

Stănescu, Heinz: Eminescu und Lenau. Temeswar 1969 Tagungsort der internationalen Lenau-Gesellschaft. In: KR 6/1969, S. 9.

Wittstock, Joachim: Zwischen Tradition und Erneurung. In: KR 13/1968, S. 8-9.

Zimann, Mihai: Die Schwaben und die Vereinigung. In: $K R$ 41/1968, S. 8. 